\title{
ON THE LARGE-SCALE STRUCTURES FORMED BY WAKES OF OPEN COSMIC STRINGS
}

\begin{abstract}
Tetsuya HARA, Shoji Morioka and Shigeru Miyoshi*
Department of physics, Kyoto Sangyo University, Kyoto 603

* Institute of Astronomy, Cambridge, UK
\end{abstract}

Large-scale structures of the universe have been variously described as sheetlike, filamentary, cellular, bubbles or spongelike.1 2) Recently cosmic strings become one of viable candidates for galaxy formation scenario ${ }^{3 \sim 10}$, and some of the large-scale structures seem to be simply explained by the open cosmic strings ${ }^{6}$.

Due to this scenario, sheets are wakes which are traces of moving open cosmic strings where dark matter and baryonic matter have accumulated.7,10) Filaments are intersections of such wakes and high density regions are places where three wakes intersect almost orthogonally $y^{6}$. The wakes formed at $t_{\text {eq }}$ become the largest surface density among all wakes, where $t_{\text {eq }}$ is the epoch when matter density equals to radiation density ${ }^{7}$, 9). Then it can be expected that the characteristic size of the sheets are $\sim 10^{2} \mathrm{Mpc} \times 10^{2} \mathrm{Mpc}$ and length of filaments are $\sim 10^{2} \mathrm{Mpc}$. It is explained that the typical surface density of sheets, line density of filaments and mass of clusters are $\sim 10^{12}\left(\mathrm{G} \mu \beta \gamma / 2 \cdot 10^{-6}\right) \mathrm{M}_{\odot} / \mathrm{Mpc}^{2}, \sim 10^{13}\left(\mathrm{G} \mu \beta \gamma / 2 \cdot 10^{-6}\right)^{2} \mathrm{M}_{\odot} / \mathrm{Mpc}$ and $\sim 10^{15}\left(\mathrm{G} \mu \beta \gamma / 2 \cdot 10^{-}\right.$ $\left.{ }^{6}\right)^{3} M_{\odot}{ }^{6}$. where $\mu$ and $\beta$ are the line density and the velocity of the string, and $\gamma=\left(1-\beta^{2}\right)^{-1 / 2}$.

If we assume that there is one open cosmic string per each horizon, then it can be explained that the typical distances among wakes, filaments and clusters are also $\sim 10^{2}$ $\mathrm{Mpc}^{11,6)}$. This model does not exclude a much more large scale structure. Open cosmic string may move even now and 
accumulate cold dark matter after its traces, however, the surface density is much smaller than the ones formed at $t_{\text {eq }}{ }^{8,9}$.

From this model, it is expected that the typical high density region will have extended features such as six filaments and three sheets and be surrounded by eight empty regions $\left(\right.$ voids) ${ }^{6}$. In this paper, we mainly concern with such structures and have made numerical simulations for the formation of such large scale structure.

We consider the Einstein-de sitter universe with $\Omega=1$, consisting of dominant dark matter $\left(\Omega_{\mathrm{d}} \simeq 0.9\right)$ and baryon matter $\left(\Omega_{b} \simeq 0.1\right)$, and $h=0.5$ is used $\left(h=H_{0} /\left(100 \mathrm{~km} \cdot \mathrm{sec} \cdot \mathrm{Mpc}^{-1}\right)\right.$ and $\mathrm{H}_{0}$ is the Hubble constant at present). Near the sheet, the accumulated dark matter and baryon matter may be fragmented due to the plane instability and objects of mass of order $\sim 10^{7} \mathrm{M}_{\odot}$ are formed around $\mathrm{z}=10^{2}$ when $\mathrm{H}_{2}$ cooling becomes effective ${ }^{7}$. For the evolution of the massive star within such objects, the universe becomes again lumonous (flare up $)^{8)}$. Due to the explosion of such massive stars will form the plane shock waves and later the shock wave will accumulate the baryon matter like snow plough. Through fragmentations of such plane, more massive secondary objects will be formed and so ${ }^{81}$.

Through such mechanism, galaxies will be formed after $1+z \simeq 7$ when the cooling time of inverse Comption by background radiation becomes longer than the expansion time of the universe. The detailed physical process through which galaxies are formed including heating and cooling process of interwake and near wake medium are now in preparation. The decrease of velocity difference between baryon objects and dark matter are discussed and due to the calculations, the velocity difference decrese rapidly if the velocity difference is smaller than the critical value ${ }^{12)}$. For simplicity, we just assumed that galaxies are formed at $1+z \simeq 7$ near wakes and follow the motion of dark matter.

Calculations are performed with $32^{3}$ particles and $32^{3}$ cells in a cube with comoving length $L=40 \mathrm{~h}^{-1} \mathrm{Mpc}$. Dark matter and galaxies are follwed by $N$-body code using the PM method with the CIC mass assignment scheme ${ }^{131}$. 
The constant density surface of dark matter where the wake is in the $x-y$ plane is shown in Fig. la. The characteristic thickness of the dark matter is given as $\mathrm{h} \sim 2 .\left(\left(1+z_{i}\right) / 6.10^{3}\right)^{1 / 2}(1+z)^{-2}\left(G \mu \beta \gamma / 10^{-6}\right) M p c^{8)}$ and such wake could be extend $10^{2} \times 10^{2} \mathrm{Mpc}^{2}$ or so. The intersection of two wakes are shown for its distribution of dark matter in Fig. $2 a$. The cone diagram ( velocity versus right ascension maps) for the galaxies are also shown in Fig. $2 b$. The case for orthogonal intersections of three wake is calculated and the distribution of the dark matter around the high density regions and cone diagrams are displayed in Figs. 3. The observation by Geller et $a 1^{11}$ is also shown in Fig. 3d.

The patterns in the cone diagram of Figs. 3 could be almost due to the combination of wall nature distributions of galaxies and slice observation. The filamentary or sheetlike distribution of galaxies around rich cluster could be checked by many slice observation.

It is difficult to estimate to what extent this model is fit to observations further than the impression of the similarity between the two graphs of Figs. $3 \mathrm{c}$ and $3 \mathrm{~d}$. Due to the impression, however details are different, it is not so bad correpondence between calculations and observations.

From the inspection of Figs. 2 and 3 , the Eilamentary structure is the slice of the wall( wake). As galaxies in the wall move into the center, one wall structure, in the redshift plane, becomes discontinuous line at the center.

It seems a little bit difficult to find out 6 filaments from the slice of CfA redshift survey by Gellar et all). Then, Coma cluster must be explained by one sheet and some instability. It could also be considerd that the wakes may not pass through orthogonally or loop of cosmic string might contribute for the formation of Coma cluster.

In general, the wall structure is rather characteristic for our calculation. To make comparison with calculations to observations in detail, we must understand much more the mechanism of galaxiy formation. 
It could be concluded that the scenario of open cosmic string with cold dark matter seems a one of good candidate for understanding the observed large scale structure of galaxies and this scenario could be enough falsifiable by observation.

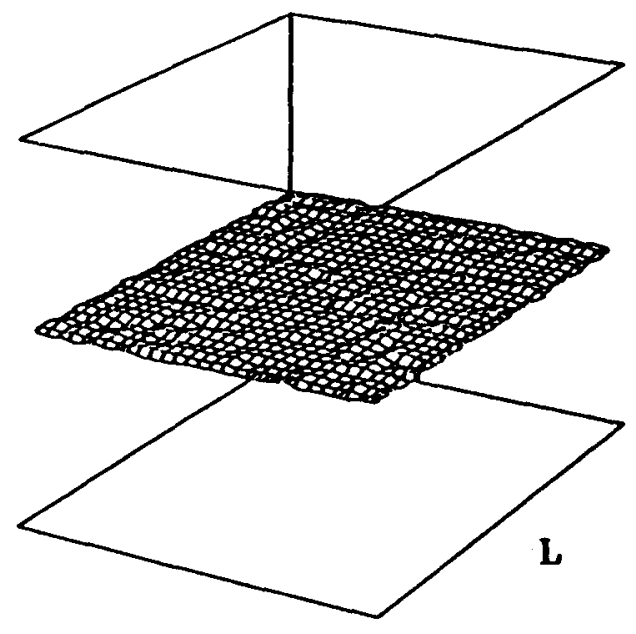

a)

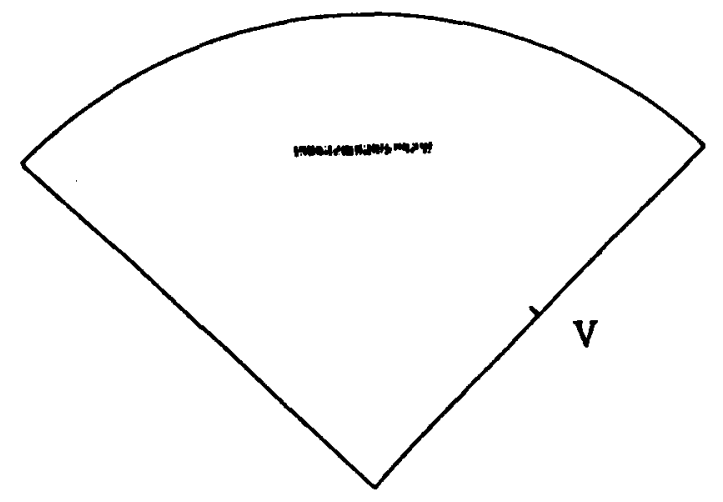

b)

Fig. 1

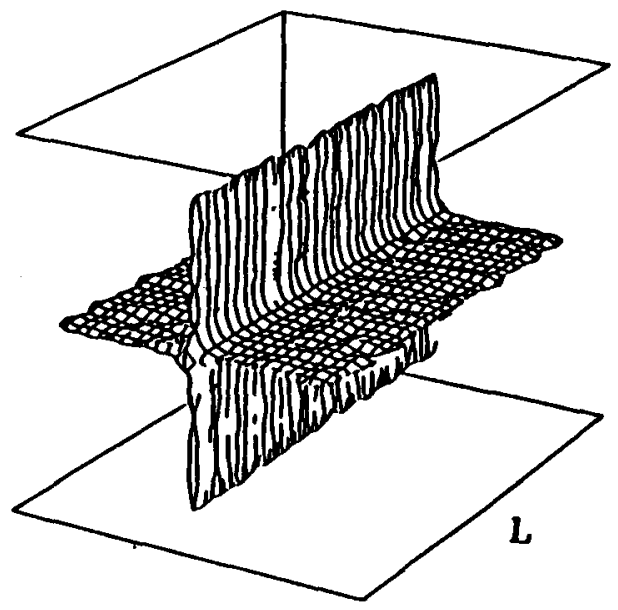

a)

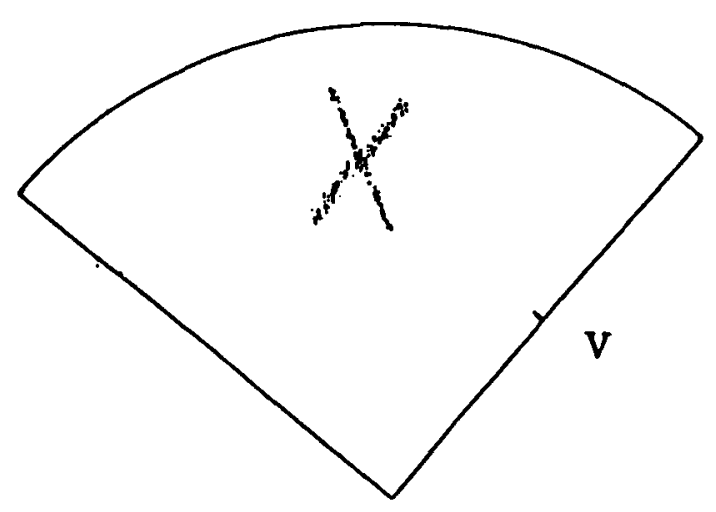

b)

Fig. 2 

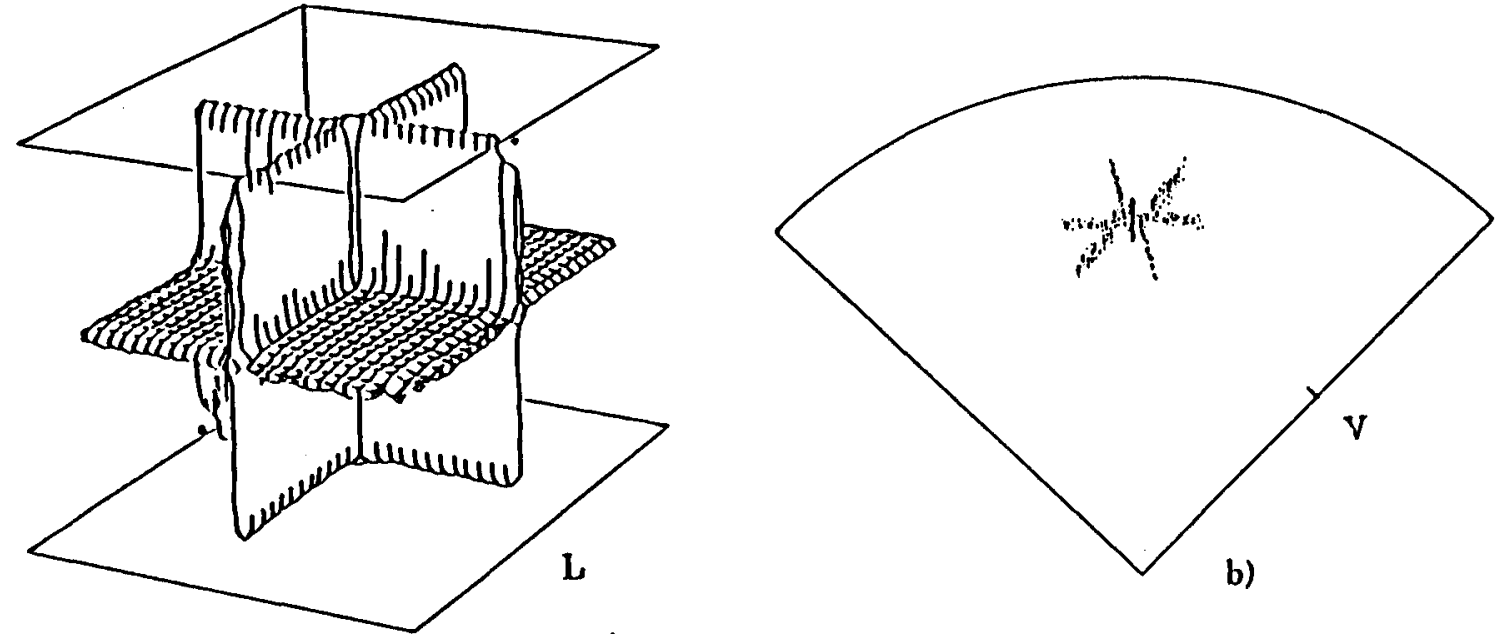

a)

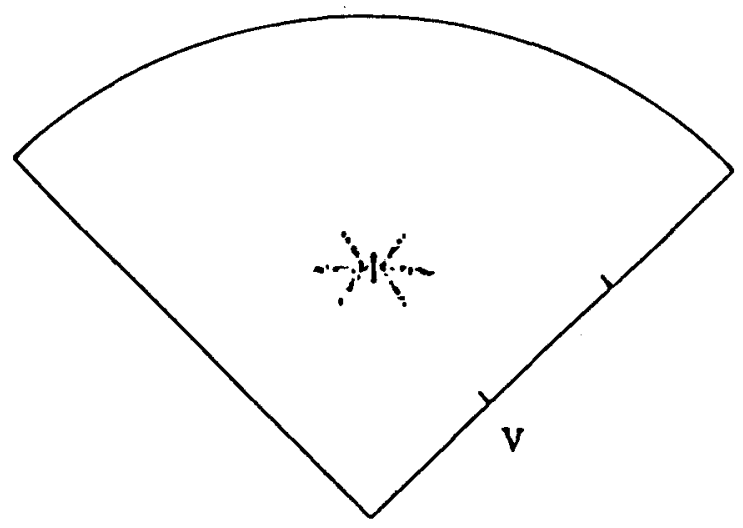

c)

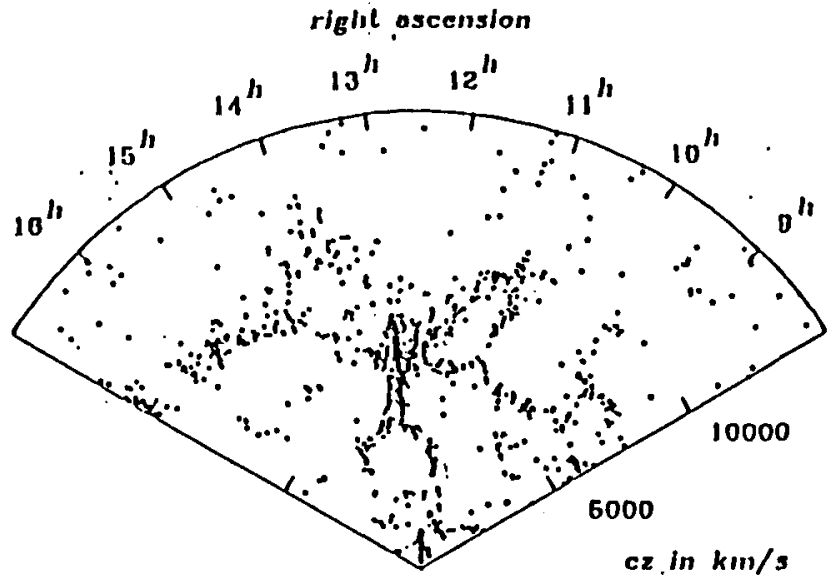

d)

\section{REFERENCES}

1) V. de Lapparent, M. J. Geller and J. P. Huchra, Agtrophys. J. 302(1986), LI.

2) M. P. Haynes and R. Glovanel11, Agtrophys. J. 306 (1986), L55.

3) A. Vilenkin, Phys. Rep. 121 (1985),263.

4) J. Sl1k and A. Vilenkin, Phys, Rev. Lett.53(1984),1700.

5) T. Vachaspatl, Phys. Rev. Lett, 57(1986), 1655.

6) T. Hara and S. Miyoshl, Prog. Theor, Phys. 81(1989), 1187.

7) T. Hara and S. Miyosh1, Prog. Theor. Phys. 77(1987), 1152.

B) T. Hara and S. Miyosh1, Prog. Theor. Phys. 78(1987), 1081.

9) A. Stebblns et. al. Astrophys. J. 322(1987), 1.

10) M. J. Rees, Mon. Not.R. Astron. Soc. 222(1986), 27p.

11) A. Albrecht and N. Turok, Phys, Rev. Lett. 54(1985), 1868.

12) T. Hara and S. Miyoshl, preprint

13) R.W.Hockney and J.W.Eastwood, "Computer Simulation Using Particles"( 1981, MacGraw Hill, New York). 